\title{
Lipid Testing Trends in the US Before and After the Release of the 2013 Cholesterol Treatment Guidelines
}

This article was published in the following Dove Press journal: Clinical Epidemiology

\author{
Sara N Levintow $\mathbb{1 D}^{1,2}$ \\ Stephanie R Reading ${ }^{3}$ \\ Bradley C Saul (D) \\ Ying Yu (1) \\ Diane Reams' \\ Leah J McGrath' \\ Kiran Philip ${ }^{3}$ \\ Paul J Dluzniewski ${ }^{3}$ \\ M Alan Brookhart ${ }^{1,4}$ \\ 'NoviSci, Inc, Durham, NC, USA; \\ ${ }^{2}$ Department of Epidemiology, University \\ of North Carolina, Chapel Hill, NC, USA; \\ ${ }^{3}$ Center for Observational Research, \\ Amgen, Thousand Oaks, CA, USA; \\ ${ }^{4}$ Department of Population Health \\ Sciences, Duke University, Durham, \\ NC, USA
}

Background: The 2013 ACC/AHA cholesterol treatment guidelines removed the recommendation to treat adults at risk of cardiovascular disease to goal levels of low-density lipoprotein cholesterol (LDL-C). We anticipated that the frequency of LDL-C testing in clinical practice would decline as a result. To test this hypothesis, we evaluated the frequency of LDL-C testing before and after the guideline release.

Methods: We used the MarketScan ${ }^{\circledR}$ Commercial and Medicare Supplemental claims data (1/1/2007-12/31/2016) to identify four cohorts: 1) statin initiators (any intensity), 2) highintensity statin initiators, 3 ) ezetimibe initiators, and 4) patients at very high cardiovascular risk ( $\geq 2$ hospitalizations for myocardial infarction or ischemic stroke, with prevalent statin use). Rates of LDL-C testing by calendar year quarter were estimated for each cohort. To estimate rates in the absence of a guideline change, we fit a time-series model to the preguideline rates and extrapolated to the post-guideline period, adjusting for covariates, seasonality, and time trend.

Results: Pre- and post-guideline rates (LDL-C tests per 1,000 persons per quarter) were 248 and 235, respectively, for 3.9 million statin initiators; 263 and 246 for 1.3 million highintensity statin initiators; 277 and 261 for 323,544 ezetimibe initiators; and 180 and 158 for 42,108 very high-risk patients. For all cohorts, observed post-guideline rates were similar to model-predicted rates. On average, the difference between observed and predicted rates was 8.5 for patients initiating any statin; 2.6 for patients initiating a high-intensity statin; 11.4 for patients initiating ezetimibe, and -0.5 for high-risk patients.

Conclusion: We observed no discernible impact of the release of the 2013 ACC/AHA guidelines on LDL-C testing rates. Rather, there was a gradual decline in testing rates starting prior to the guideline change and continuing throughout the study period. Our findings suggest that the guidelines had little to no impact on use of LDL-C testing.

Keywords: low-density lipoprotein cholesterol, statin, ezetimibe, cardiovascular disease, guideline adherence, database, epidemiology

\section{Introduction}

In 2013, the American College of Cardiology and the American Heart Association (ACC/AHA) released updated guidelines on cholesterol treatment for adults at risk of atherosclerotic cardiovascular disease (ASCVD). ${ }^{1}$ Notably, the guidelines removed the recommendation to treat patients to goal levels of low-density lipoprotein cholesterol (LDL-C). This departure from the treat-to-target paradigm was due to insufficient evidence for titrating cholesterol-lowering therapy to specific
Correspondence: Sara $N$ Levintow NoviSci, Inc, PMB 218, 20I W Main St, Ste 200, Durham, NC 2770I

Email slevintow@novisci.com 
LDL-C goals. ${ }^{1,2}$ Instead, the guidelines recommended a fixed-dose statin therapy at an appropriate intensity (based on four statin benefit groups) without ongoing monitoring of LDL-C goals. As a result of these updated guidelines, the frequency of follow-up LDL-C testing for patients on lipid-lowering therapies was expected to decline from the pre- to the post-2013 guideline eras. ${ }^{3}$ However, the degree to which LDL-C testing frequency has changed is not well understood.

Given that the 2013 ACC/AHA guidelines were anticipated to greatly expand statin use in the United States, ${ }^{3}$ studies have focused on changes in the use of lipidlowering therapies, finding mixed evidence for impact of the guidelines. Prior to the guideline release, inadequate statin dosing, low adherence to therapy, and high rates of discontinuation had been observed for patients at risk of ASCVD events, ${ }^{4}$ as well as suboptimal statin use and LDL-C monitoring after hospitalization for myocardial infarction (MI). ${ }^{5}$ Following the guideline release, some studies have shown higher rates of initiation of moderateto high-intensity statin therapy, relative to rates in the year prior to the guidelines; ${ }^{6}$ improved adherence to statins has also been observed. ${ }^{7}$ However, other studies evaluating the impact of the guideline release have found no appreciable change $^{8}$ or only modest increases in statin use., Comparing statin use in the year prior to the year following the guideline release, two studies found that moderateto high-intensity statin use increased by 3-5 percentage points among commercially insured adults with ASCVD $^{9}$ and patients at cardiology practices with elevated cholesterol, ASCVD, or diabetes. ${ }^{10}$ These two studies are among the few to evaluate the frequency of LDL-C testing; in both studies, the average number of LDL-C tests per patient went unchanged from the year before to the year after the guideline release.

Therefore, the objective of our study was to investigate LDL-C testing frequency prior to and following the release of the 2013 ACC/AHA guidelines using real-world data from a large commercially insured patient population. To build on previously published studies, we estimated rates of LDL-C testing from 2008 to 2016, rather than simply comparing the counts of tests in the year before and year after the guideline release. Unlike prior studies, we modeled the change in rates due to the guideline change, which enabled our study to control for covariates and seasonality. In addition, we broadened the patient population to all adults initiating a statin or ezetimibe, not only those with diagnosed ASCVD. Our study also examined demographic and clinical predictors of follow-up LDL-C testing following initiation of a statin, ezetimibe, or cardiovascular hospitalization. We hypothesized that LDL-C testing had declined since the 2013 guideline change; however, patients with greater or more severe cardiovascular risk predictors (eg, history of ASCVD events) would receive more frequent LDL-C testing compared to those with fewer or less severe cardiovascular risk predictors.

\section{Methods}

\section{Data Source and Study Population}

This study used data from the MarketScan ${ }^{\circledR}$ Commercial Claims and Encounters and Medicare Supplemental administrative health insurance claims databases (IBM Corp.) to identify patients initiating a statin or ezetimibe, and those at high cardiovascular risk. The MarketScan database captures healthcare claims data for privately insured individuals $(<65$ years) and individuals with Medicare Supplemental insurance ( $\geq 65$ years). Data were available on inpatient and outpatient diagnoses, procedures, and medications, which were identified using codes from the International Classification of Diseases, clinical modification, ninth (ICD-9-CM) and tenth revisions (ICD-10-CM), Healthcare Common Procedure Coding System (HCPCS) codes, Current Procedural Terminology (CPT) codes, and National Drug Codes (NDC). Patients were identified between 01/01/2008 and $12 / 31 / 2015$, with data available from $01 / 01 / 2007$ to determine eligibility criteria and covariate information. Data were available for follow-up through 12/31/2016.

The study population consisted of four cohorts: 1) patients who initiated a statin (regardless of intensity), 2) patients who initiated a high-intensity statin, 3) patients who initiated ezetimibe, and 4) patients at very high cardiovascular risk (defined as $\geq 2$ hospitalizations for myocardial infarction (MI) and/or ischemic stroke (IS) and prevalent moderate- or high-intensity statin use). Statins and ezetimibe were identified using NDCs. MI hospitalizations were identified using inpatient claims with ICD-9 410.xx (excluding 410.x2) or ICD-10 I21.xx, I22.x; IS hospitalizations were identified using inpatient claims with ICD-9 433.x1, 434.x1 (excluding 434.11) or ICD-10 I63.xxx (excluding I63.4xx). Patients entered the study population at the time of the index event (date of new statin use, new ezetimibe use, or most recent MI or IS hospitalization discharge) if they were at least 21 years of age and had continuous health insurance coverage over the 
prior 365 days. Patients could contribute data to more than one cohort.

Each patient was followed for occurrences of LDL-C testing starting the day after their index event. LDL-C tests were identified using CPT and HCPCS codes associated with inpatient or outpatient claims. Follow-up continued until the earliest of: nonadherence to statin or ezetimibe (45 day-gap since end date of last recorded prescription), disenrollment from insurance (all patients), or 12/31/2016 (end of study). Appendix 1 contains LDL-C testing codes and statin intensity classifications used in this study.

\section{Statistical Analysis}

For each cohort, we evaluated the distribution of patient covariates using all available data leading up to and including the date of the index event. Covariates included demographics (age, sex, geographic region, insurance benefit plan), history of ASCVD events (MI, IS, aneurysm, carotid/vertebral/basilar stenosis, carotid endarterectomy, cerebrovascular disease, coronary atherosclerosis/angina/ old MI, CABG/PCI, carotid/vertebral/basilar stenting, endovascular stent graft, peripheral vascular disease, peripheral artery disease, transient ischemic attack, unstable angina), other cardiovascular conditions and comorbidities (end-stage renal disease or hemodialysis, chronic kidney disease, heart failure, diabetes, cancer, cognitive impairment, hypertension, dyslipidemia/hyperlipidemia, muscle events, obesity, chronic obstructive pulmonary disease or asthma, hepatic disorders), markers of frailty (oxygen use, wheelchair, hospital bed, rehabilitation services, difficulty walking), concomitant lipid-lowering medication, and prior LDL-C testing. All covariates were identified using algorithms of inpatient and outpatient ICD-9-CM or ICD10-CM diagnosis or procedure codes, CPT or HCPCS procedure codes, and/or NDC. These algorithms were developed by our study team using a combination of a priori subject matter knowledge and previously published algorithms. ${ }^{11-13}$

We estimated the rate of LDL-C testing for each quarter throughout the study period (eg, 01/01/2008-03/31/ 2008, 04/01/2008-06/30/2008, through 10/01/2016-12/31/ 2016). Rates were estimated as the count of tests over the person-time per quarter using a Poisson model with a variance inflation factor. We also included a model term indicating whether the quarter took place in the preor post-2013 guideline release period; this allowed us to estimate the overall pre-guideline rate and the overall postguideline rate, in addition to each quarterly rate. Models were run separately for each of the four cohorts to obtain rate estimates specific to each patient population.

We then used a time-series model to estimate the change in quarterly LDL-C testing rates following the 2013 guideline release, accounting for differences in patient covariates over time. We fit the same Poisson model to quarterly rates prior to the 2013 guideline change and then extrapolated this model to the post-guideline time period to predict LDL-C testing rates in the absence of a guideline change. We estimated the change in testing rates by quarter as the difference between these model predicted rates and the observed rates corresponding to each quarter starting in 01/01/2014-03/31/2014 through $10 / 01 / 2016-12 / 31 / 2016$. The quarter when the guideline change occurred (10/01/2013-12/31/2013) was treated as the last pre-guideline quarter. The time-series model included all baseline covariates, cumulative quarters in the cohort, seasonality terms, and a linear term for time. These analyses were also stratified by subgroups to understand if changes in LDL-C testing rates varied for different types of patients. Subgroups were defined by age group ( $<65$ years, 65-75, >75 years), US geographic region (Midwest, Northeast, South, West), history of ASCVD event (any past event, no past event, event in last 6 months), and history of LDL-C testing (any prior testing, no prior testing). In addition, we assessed the sensitivity of findings to potential misclassification of statin initiation. All analyses were repeated after restricting to patients with new use of a generic statin (given that patients initiating a brand-name statin may be misclassified as new users if they had previously used brand-name samples).

Finally, in addition to estimating changes in LDL-C testing, we evaluated predictors of LDL-C testing by cohort. All baseline covariates were evaluated as predictors in the Poisson model, which estimated rate ratios for each predictor's association with quarterly LDL-C testing rates over follow-up. This study was a secondary analysis of deidentified data and was approved by the Chesapeake Institutional Review Board, which ensured that all data accessed for the study complied with patient data privacy regulations. All statistical analyses were performed using $\mathrm{R}$ software, version 3.5.2 (see Appendix 2 for R packages used in the analysis). ${ }^{14}$

\section{Results}

Among 15,607,733 patients in the database during the study period, the following qualified for the four study cohorts (Figure 1): 3,874,048 patients initiated a statin 


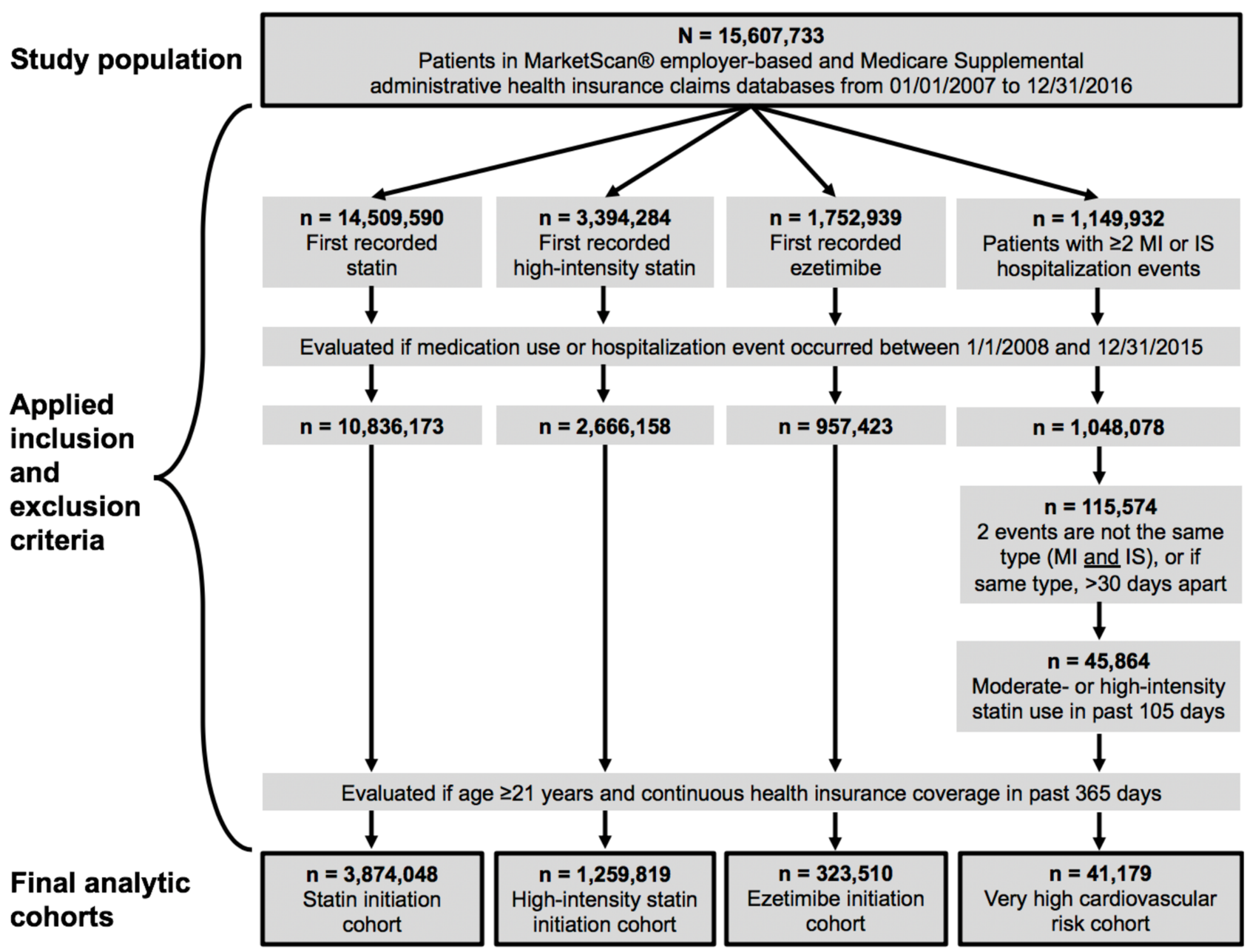

Figure I Study flow chart. Inclusion and exclusion criteria were applied to 15,607,733 patients in the MarketScan ${ }^{\circledR}$ and Medicare Supplemental claims databases to select the four study cohorts.

(regardless of intensity); 1,259,819 initiated a highintensity statin; 323,510 initiated ezetimibe; and 41,179 were at very high cardiovascular risk (referred to as "high risk" hereafter). Table 1 shows the characteristics of patients in the four cohorts. Most patients initiating statins or ezetimibe were aged 50-64 years, while highrisk patients were older ( $\geq 65$ years). Patients initiating a high-intensity statin or at high risk were more likely to be male (58-59\%) compared to patients initiating ezetimibe or a statin of any intensity (51-52\%). By study design, all high-risk patients had a history of ASCVD events; in contrast, history of ASCVD events was observed for $15 \%$ and $33 \%$ of patients initiating any statin or a high-intensity statin, respectively, and $25 \%$ of patients initiating ezetimibe. The prevalence of comorbidities such as hypertension, hyperlipidemia, chronic obstructive pulmonary disease, diabetes, and cancer were greater in the high-risk cohort, compared to patients initiating statins or ezetimibe. More high-risk patients had a history of frailty (76\%) and cognitive impairment (37\%), compared to considerably lower levels in the other cohorts $(25-31 \%$ for frailty and $2-3 \%$ for cognitive impairment). Approximately $80 \%$ of patients initiating statins or ezetimibe had a history of prior LDL-C testing, compared to $64 \%$ of high-risk patients.

All rates are reported as the number of LDL-C tests per 1,000 persons per calendar year quarter. The overall preand post-guideline unadjusted rates were 270 and 256, respectively, for patients initiating any statin; 276 and 256 for patients initiating a high-intensity statin; 288 and 281 for patients initiating ezetimibe; and 177 and 155 for high-risk patients. When extrapolating pre-guideline rate estimates to the post-guideline period, the observed postguideline rates of LDL-C testing were similar to or slightly higher than the model-predicted rates (Figure 2). On average, the difference between observed and predicted rates 
Table I Characteristics of Study Cohort Patients Using All Available Data Leading Up to and Including the Date of the Index Event (Medication Initiation or CVD Hospitalization Event)

\begin{tabular}{|c|c|c|c|c|}
\hline Baseline Characteristic (\%) & $\begin{array}{l}\text { Any Statin } \\
\text { Initiation, } \\
n=3,874,048\end{array}$ & $\begin{array}{l}\text { High-Intensity Statin } \\
\text { Initiation, } \\
n=1,259,819\end{array}$ & $\begin{array}{l}\text { Ezetimibe } \\
\text { Initiation, } \\
n=323,510\end{array}$ & $\begin{array}{l}\text { Very High CVD } \\
\text { Risk, } \\
\text { n = 41, } 179\end{array}$ \\
\hline \multicolumn{5}{|l|}{ Demographics } \\
\hline \multicolumn{5}{|l|}{ Age group } \\
\hline $18-39$ & 7.7 & 4.4 & 4.2 & 0.7 \\
\hline $40-49$ & 20.6 & 15.7 & 14.6 & 4.3 \\
\hline $50-64$ & 54.7 & 55.9 & 58.0 & 30.2 \\
\hline $65+$ & I7.I & 23.9 & 23.2 & 64.8 \\
\hline \multicolumn{5}{|l|}{ Sex } \\
\hline Male & 51.9 & 59.4 & 51.3 & 58.1 \\
\hline Female & 48.1 & 40.6 & 48.7 & 41.9 \\
\hline \multicolumn{5}{|l|}{ Region* } \\
\hline Northeast & 15.4 & 14.8 & 14.1 & 19.5 \\
\hline North Central & 25.0 & 25.4 & 26.5 & 34.5 \\
\hline South & 41.9 & 42.4 & 44.5 & 31.9 \\
\hline West & 14.7 & 15.0 & 12.9 & 13.3 \\
\hline \multicolumn{5}{|l|}{ Type of insurance benefit plant } \\
\hline Comprehensive & 7.3 & 10.8 & 9.8 & 32.5 \\
\hline HMO & 13.4 & 13.9 & 11.3 & 13.8 \\
\hline PPO & 61.2 & 58.0 & 63.7 & 43.3 \\
\hline Other & 14.6 & 14.6 & 11.7 & 8.3 \\
\hline \multicolumn{5}{|l|}{ History of ASCVD events } \\
\hline Any ASCVD event & 15.3 & 32.9 & 25.4 & 100.0 \\
\hline \multicolumn{5}{|l|}{ Most recent time of ASCVD event $\ddagger$} \\
\hline$<$ I month & 9.2 & 19.7 & 10.8 & 99.1 \\
\hline I-6 months & 3.4 & 7.7 & 8.8 & 0.9 \\
\hline $7-12$ months & 1.3 & 2.7 & 3.1 & 0.0 \\
\hline$>12$ months & 1.4 & 2.8 & 2.7 & 0.0 \\
\hline No ASCVD event & 84.8 & 67.1 & 74.6 & 0.0 \\
\hline Aneurysm & 0.8 & 1.5 & I.I & 8.0 \\
\hline Carotid/vertebral/basilar stenosis & 2.0 & 4.4 & 3.4 & 38.4 \\
\hline Carotid endarterectomy & 0.2 & 0.5 & 0.4 & 5.8 \\
\hline Cerebrovascular disease & 3.9 & 7.4 & 5.3 & 66.0 \\
\hline $\begin{array}{l}\text { Coronary atherosclerosis/angina/old } \\
\text { MI }\end{array}$ & 9.6 & 25.2 & 20.4 & 78.2 \\
\hline $\mathrm{CABG} / \mathrm{PCl}$ & 4.0 & 11.5 & 6.5 & 41.5 \\
\hline Carotid/vertebral/basilar stenting & 0.0 & 0.1 & 0.1 & 1.8 \\
\hline Endovascular stent graft & 0.0 & 0.1 & 0.1 & 0.6 \\
\hline Ischemic stroke & 2.2 & 3.7 & 1.7 & 62.1 \\
\hline Myocardial infarction & 3.8 & 10.0 & 4.9 & 71.0 \\
\hline Peripheral vascular disease & 2.4 & 5.1 & 4.1 & 32.3 \\
\hline Peripheral artery disease & 0.4 & 1.0 & 0.8 & 7.4 \\
\hline Transient ischemic attack & 1.6 & 2.5 & 1.5 & 32.5 \\
\hline Unstable angina & 2.1 & 5.5 & 3.5 & 30.7 \\
\hline
\end{tabular}

(Continued) 
Table I (Continued).

\begin{tabular}{|c|c|c|c|c|}
\hline Baseline Characteristic (\%) & $\begin{array}{l}\text { Any Statin } \\
\text { Initiation, } \\
n=3,874,048\end{array}$ & $\begin{array}{l}\text { High-Intensity Statin } \\
\text { Initiation, } \\
n=1,259,819\end{array}$ & $\begin{array}{l}\text { Ezetimibe } \\
\text { Initiation, } \\
n=323,510\end{array}$ & $\begin{array}{l}\text { Very High CVD } \\
\text { Risk, } \\
\text { n = 41,179 }\end{array}$ \\
\hline \multicolumn{5}{|c|}{ Other comorbidities and medication use } \\
\hline $\begin{array}{l}\text { End-stage renal disease or } \\
\text { hemodialysis }\end{array}$ & 0.3 & 0.5 & 0.3 & 6.4 \\
\hline Chronic kidney disease, all stages & 4.4 & 8.0 & 6.1 & 46.9 \\
\hline Heart failure & 2.2 & 4.4 & 3.4 & 38.7 \\
\hline Diabetes & 20.4 & 30.6 & 27.0 & 52.9 \\
\hline Cancer§ & 5.0 & 6.3 & 5.7 & 17.6 \\
\hline Cognitive impairment & 2.0 & 2.8 & 1.8 & 36.5 \\
\hline Hypertension & 44.3 & 57.4 & 52.3 & 91.4 \\
\hline Dyslipidemia/hyperlipidemia & 13.9 & 23.2 & 25.0 & 34.3 \\
\hline $\begin{array}{l}\text { Muscle events (rhabdomyolysis/ } \\
\text { myositis) }\end{array}$ & 2.9 & 3.3 & 4.6 & 7.0 \\
\hline Obesity & 2.2 & 3.1 & 2.1 & 7.1 \\
\hline COPD or asthma & 6.9 & 9.0 & 8.0 & 26.9 \\
\hline Hepatic disorders & 0.8 & 1.0 & I.I & 4.5 \\
\hline Frailty & 25.2 & 31.3 & 29.5 & 76.3 \\
\hline $\begin{array}{l}\text { Concomitant lipid lowering } \\
\text { medication }\end{array}$ & 8.6 & 15.3 & 18.9 & 18.2 \\
\hline Prior LDL-C testing & 78.9 & 79.6 & 80.0 & 63.8 \\
\hline
\end{tabular}

Notes: *Percentages do not sum to 100 due to missing data ( $\leq 3 \%$ for all cohorts); ${ }^{\dagger}$ Percentages do not sum to 100 due to missing data ( $\leq 4 \%$ for all cohorts). Other insurance types were exclusive provider organization (EPO), point-of-service plan (POS), POS with capitation, consumer-driven health plan (CDHP), and high-deductible health plan (HDHP); ${ }^{\ddagger}$ The very high CVD risk group qualified for the study cohort at the time of discharge from the most recent MI or IS hospitalization. Therefore, by definition, the most recent ASCVD event for all patients in this cohort occurred a short period of time before their hospitalization discharge. In rare instances (<1\%), the most recent MI or IS event occurred over one month prior to study entry at discharge, suggesting a long hospitalization; ${ }^{\S}$ The cancer variable excluded non-melanoma skin cancer.

Abbreviations: $\mathrm{HMO}$, health maintenance organization; PPO, preferred provider organization; CABG/PCl, coronary artery bypass grafting or percutaneous coronary intervention; COPD, chronic obstructive pulmonary disease.

was 8.5 for patients initiating any statin; 2.6 for patients initiating a high-intensity statin; 11.4 for patients initiating ezetimibe, and -0.5 for high-risk patients (Figure 3 ).

When evaluating possible predictors of LDL-C testing rates, a history of LDL-C testing (prior to index) was the strongest predictor of follow-up testing observed during the study period (rate ratios ranged between 2.5 and 3.4 across cohorts). Figure 4 shows the rate ratios for other baseline covariates (all 95\% CI are extremely narrow due to large sample size). Age was a strong predictor of testing, with patients at older ages ( $\geq 65$ years) receiving testing at approximately half of the rate observed for younger patients. In general, history of ASCVD events predicted somewhat higher testing rates, although these associations varied by study cohort. Several comorbidities and medication use were associated with slight increases in testing rates, including diabetes (all rate ratios 1.2 across cohorts), dyslipidemia/hyperlipidemia (rate ratios 1.1), concomitant lipid-lowering medication (rate ratios 1.1), and chronic kidney disease (rate ratios 1.0-1.1). Cognitive impairment was associated with small decreases in testing rates (rate ratios 0.9 ).

When we stratified the time-series analyses by subgroups for age, geographic region, history of ASCVD events, and history of LDL-C testing, our findings were generally consistent with the unstratified models (see Appendix 3 for full study report with subgroup analyses). We also repeated the time-series analysis after restricting patients to those with a new use of a generic statin (excluding those initiating brand-name statins) and did not observe any appreciable change in results (Appendix 3).

\section{Discussion}

Using real-world data from a large commercially insured patient population, we observed no discernible impact of the release of the 2013 ACC/AHA guidelines on LDL-C testing rates. Rather, we reported a gradual decline in LDL-C testing rates starting prior to the guideline change 


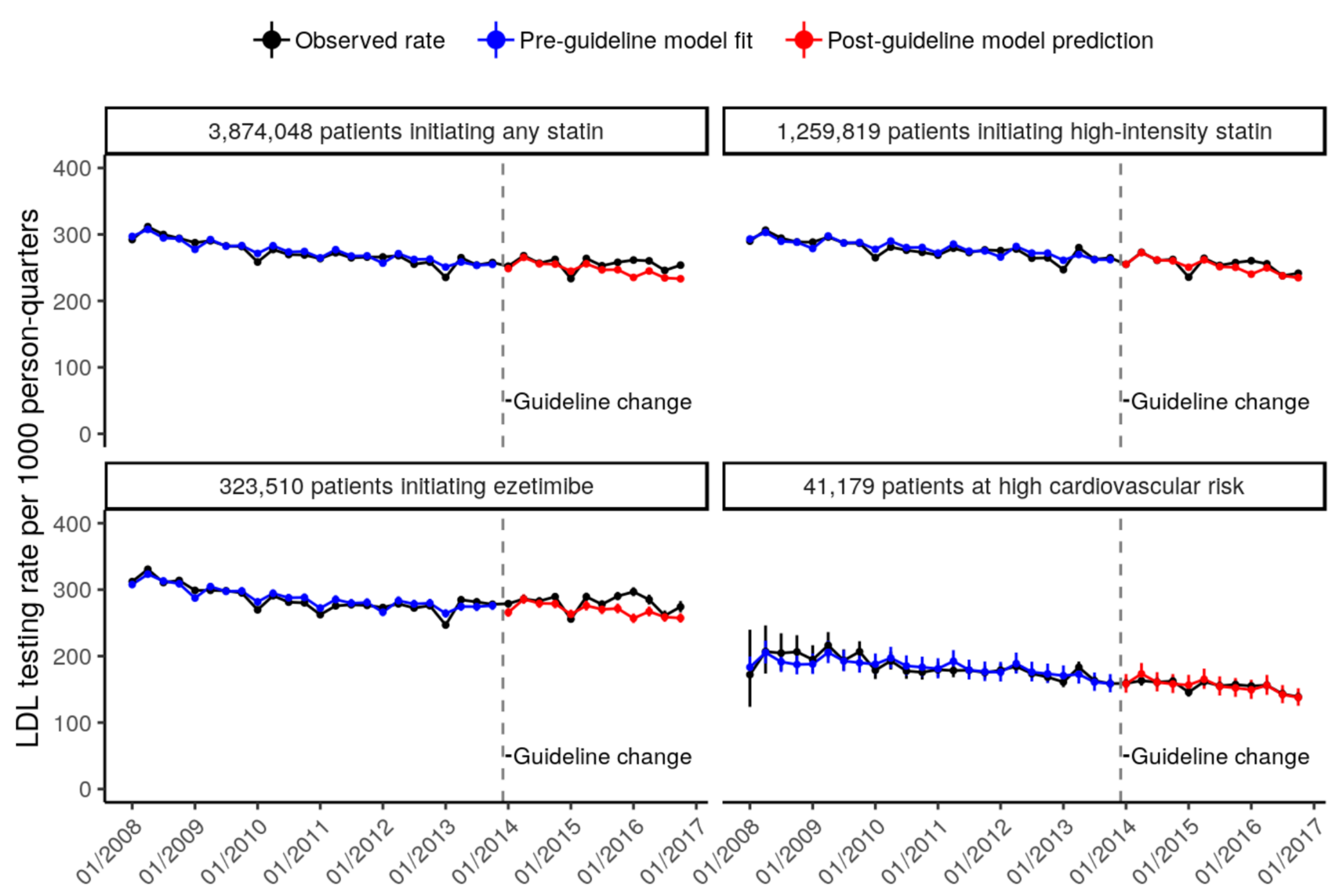

Figure 2 LDL-C testing rates (with $95 \% \mathrm{Cl}$ ) per I,000 persons per calendar year quarter from 2008 to 2016, by study cohort. The unadjusted observed rates are plotted in black; the time-series model fit to the pre-guideline data is plotted in blue; and the model predictions extrapolated to the post-guideline period are plotted in red. The dashed line corresponds to the date of the guideline change, and the quarter during which the guideline change occurred (I0/0I/20I3-I2/3I/20I3) was treated as the last pre-guideline quarter.

and continuing throughout the study period (2008 to 2016). This trend was observed across four study cohorts representing diverse patient populations: patients initiating statins at any intensity, patients initiating a high-intensity statin, patients initiating ezetimibe, and patients at very high cardiovascular risk. We used time-series models to extrapolate rate estimates (based only on pre-guideline data) to the postguideline period. Across study cohorts, we observed that post-guideline rates of LDL-C testing were similar to or slightly higher than the model-predicted rates. LDL-C testing rates were substantially lower for high-risk patients, but this was observed across the study period (rather than indicating any effect of the guideline change). Our results also remained consistent across a variety of subgroup and sensitivity analyses. Despite the departure from the treat-to-target paradigm in the 2013 guidelines, we found no evidence to support our hypothesis that LDL-C testing declined following this guideline change.

To our knowledge, only two prior studies evaluated changes in the frequency of LDL-C testing resulting from the 2013 guideline change. ${ }^{9,10}$ Both found that the average number of LDL-C tests per patient went unchanged from the year before to the year after the 2013 guideline release (approximately 1 test per year among patients with ASCVD from the Optum claims database; ${ }^{9} 0.5$ test per year among patients with elevated cholesterol, ASCVD, or diabetes from cardiology practice data ${ }^{10}$ ). Our study broadened the patient population under study (including adults initiating statins or ezetimibe, without diagnosed ASCVD) and expanded follow-up time (2008 to 2016); this allowed observation of a gradual decline in LDL-C testing rates across diverse patient groups. However, consistent with prior work, ${ }^{9,10}$ our study found no appreciable change in LDL-C testing rates following the 2013 guideline change, outside of the ongoing decline in testing.

We also examined demographic and clinical predictors of LDL-C testing in our patient cohorts (following initiation of a statin, ezetimibe, or cardiovascular hospitalization). Unsurprisingly, a history of prior LDL-C testing was the strongest predictor of future testing; across study cohorts, 


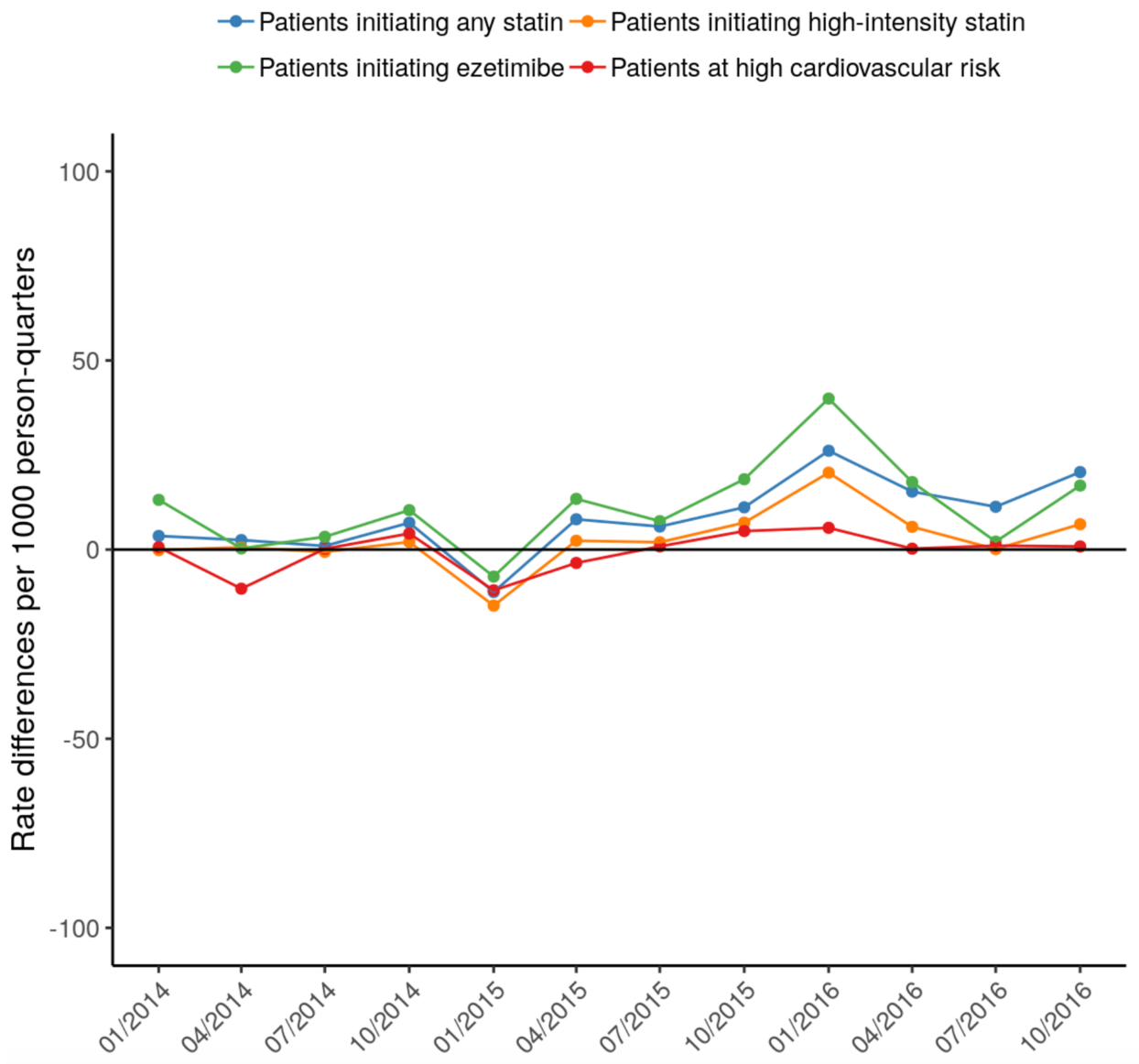

Figure 3 Differences between observed rates and model-predicted rates of LDL-C tests per I,000 persons per calendar year quarter, by study cohort. Differences were taken by subtracting the model-predicted rate from the observed rate for each calendar year quarter following the guideline change.

rates for patients with a history of LDL-C testing (prior to medication initiation or cardiovascular hospitalization) were two to three times the rates of patients without a history of testing. Younger age ( $<65$ years) was also associated with higher rates of LDL-C testing, which was consistent with the higher testing rates we observed in patient cohorts initiating statins or ezetimibe (younger on average) and lower testing rates in the high cardiovascular risk cohort (older on average). History of ASCVD events and other comorbid conditions was associated with increased testing rates, although the magnitude of these associations was generally small. For the subset of patients with recent MI/IS hospitalization and prevalent statin use, LDL-C testing rates were lower than those observed for the other study cohorts. This may reflect lower levels of testing for these patients prior to hospitalization (due to unrecognized dyslipidemia or other cardiovascular risk factors) without closer monitoring implemented following hospitalization.

Although the 2013 guidelines no longer recommend titrating lipid-lowering therapy to LDL-C goals, our findings suggest that physicians continue to order LDL-C tests at rates expected had no guideline change occurred. This may be due to lack of awareness or understanding of the guidelines. In a prior study on provider understanding of the 2013 guidelines, fewer than half of providers surveyed had read the guidelines. ${ }^{15}$ It is also possible that decreased LDL-C testing preceded release of the guidelines. Evidence for abandoning specific LDL-C goals had accumulated prior to the 2013 guideline release, and some physicians may have already incorporated this evidence into their LDL-C testing practices (resulting in the gradual decline in LDL-C testing observed in years prior to 2013). In addition, physicians are faced with an overwhelming number of treatment guidelines for cholesterol management, and recommendations from different organizations may conflict. For example, the National Lipid Association recommendations from 2014 still advocate to treat to LDL-C goals. ${ }^{16,17}$ This was likely a function of the lack of consensus in the clinical community and the ensuing differences in approaches for developing each set of 


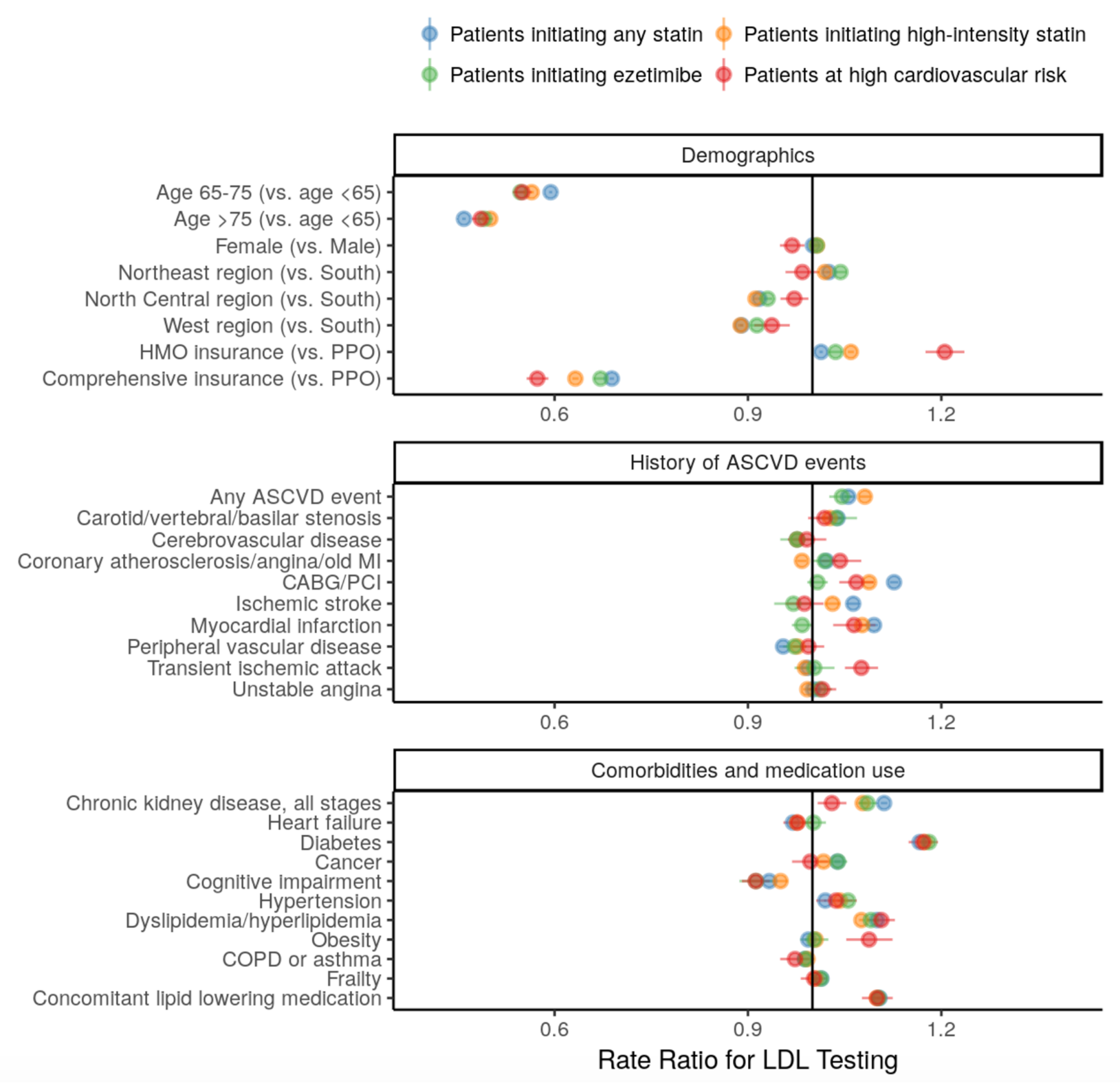

Figure 4 Rate ratios (with 95\% Cl) for LDL-C testing associated with predictor variables. Predictors included demographics, history of ASCVD events, comorbidities and medication use. Their association with LDL-C testing (following index event) was estimated separately by study cohort and was not computed if prevalence of the predictor was $<1 \%$.

guidelines (eg, reliance on randomized controlled trials for the ACC/AHA guidelines vs inclusion of evidence from observational studies in the NLA recommendations). ${ }^{17}$ Therefore, physicians' disagreement with the guidelines, or confusion over which recommendation to follow, may result in continued use of the treat-to-target approach.

Unchanged LDL-C testing rates (as observed in our study) do not necessarily indicate a lack of behavioral change in physicians in response to the guidelines. Importantly, although the 2013 guidelines removed the recommendation to treat to LDL-C goals, they do still recommend monitoring LDL-C to determine adherence to therapy and anticipated therapeutic response. One limitation of insurance claims data alone is that we cannot discern the reason for LDL-C testing. Even if physicians are no longer treating to LDL-C goals, they may order tests just as frequently to ascertain adherence and response to therapy. ${ }^{9}$ In addition, our findings represent LDL-C testing rates among adults with employer-based insurance or Medicare supplemental insurance, which may not generalize to LDL-C testing trends among all adults in the US. We also restricted the study population to patients with 
continuous health insurance coverage in the one year prior to medication initiation or MI/IS hospitalization. This restriction ensured that all study patients had at least one year of available data to define baseline covariates (to improve confounding control) and identify medication initiation (to reduce misclassification of prevalent use). Although sample sizes remained large, this exclusion may further limit the generalizability of our findings (eg, to the extent that different patterns of LDL-C testing occur among patients who change insurance plans or lose insurance coverage).

The updated AHA/ACC guidelines on cholesterol management were released in 2018 and continue to recommend ongoing LDL-C monitoring to assess therapeutic adherence and response. ${ }^{18}$ The 2018 guidelines incorporate evidence from recent clinical trials showing that the addition of nonstatin therapies (ezetimibe and proprotein convertase subtilisin/kexin type 9 inhibitors) further reduce LDL-C and the risk of cardiovascular events, compared to statin therapy alone. ${ }^{19-21}$ These recent findings support the "lower is better" hypothesis for LDL-C and may renew focus on treatment decisions driven by LDL-C levels rather than intensity of statin therapy alone. If this new evidence in the 2018 guidelines translates to increased LDL-C monitoring, the downward trends in testing observed in our study (through 2016) may gradually rise in more recent years.

\section{Conclusions}

Our findings suggest that the 2013 ACC/AHA guidelines had limited impact on LDL-C testing rates, outside of the ongoing, gradual decline. We did not find evidence that the 2013 guidelines translated to less stringent monitoring of LDL-C levels. Trends in LDL-C testing were similar for diverse groups of patients initiating statins, ezetimibe, or at very high cardiovascular risk. Across these patient cohorts, we also found modest associations between demographic and clinical variables and LDL-C testing rates, with history of prior LDL-C testing most predictive of continued follow-up testing. Due to the absence of any effect of the 2013 guidelines on LDL-C testing rates, researchers can continue to rely on routinely collected LDL-C data to study populations with ASCVD risk. Given the release of the updated 2018 cholesterol guidelines, future research is warranted to investigate current trends in LDL-C testing and implications for epidemiologic research using realworld data.

\section{Data Sharing Statement}

Study data are available for purchase from IBM Corp. Readers can request $\mathrm{R}$ code from the corresponding author.

\section{Ethics Approval}

This study was approved by the Chesapeake Institutional Review Board.

\section{Acknowledgments}

Portions of this study were presented at the 2019 American Heart Association Scientific Sessions as a poster presentation with interim findings. The poster's abstract was published in "Abstracts from the American Heart Association's 2019 Scientific Sessions" in Circulation: https://www.ahajournals.org/doi/abs/10.1161/ circ.140.suppl 1.13025.

\section{Author Contributions}

All authors made substantial contributions to conception and design, acquisition of data, or analysis and interpretation of data; took part in drafting the article or revising it critically for important intellectual content; agreed to submit to the current journal; gave final approval of the version to be published; and agree to be accountable for all aspects of the work.

\section{Funding}

This work was funded by Amgen.

\section{Disclosure}

S.N. Levintow is an employee and owns stock in NoviSci, Inc. and received doctoral training support from the National Institute on Drug Abuse (R36 DA045569), National Institute of Allergy and Infectious Disease (T32 AI070114-10), and ViiV Healthcare (pre-doctoral fellowship). S.R. Reading is an employee and owns stock in Amgen, Inc. B.C. Saul is an employee and owns stock in NoviSci, Inc. Y. Yu is an employee and owns stock in NoviSci, Inc. D. Reams is an employee and owns stock in NoviSci, Inc. L.J. McGrath is an employee and owns stock in NoviSci, Inc. K. Philip is an employee and owns stock in Amgen, Inc. P.J. Dluzniewski is an employee and owns stock in Amgen, Inc. M.A. Brookhart is an employee and owns stock in NoviSci, Inc and in the last three years has served as a scientific advisor to AbbVie, Amgen, the Brigham and Women's Hospital, Genentech, Gilead, Merck, Rockwell Medical and Vertex. He also 
reports grants from BlueCross BlueShield of North Carolina and North Carolina Department of Health and Human Services. The authors report no other conflicts of interest in this work.

\section{References}

1. Stone NJ, Robinson JG, Lichtenstein AH, et al. 2013 ACC/AHA guideline on the treatment of blood cholesterol to reduce atherosclerotic cardiovascular risk in adults. J Am Coll Cardiol. 2014;63 (25):2889-2934. doi:10.1016/j.jacc.2013.11.002

2. Hayward RA, Krumholz HM. Three reasons to abandon low-density lipoprotein targets. Circ Cardiovasc Qual Outcomes. 2012;5(1):2-5. doi:10.1161/CIRCOUTCOMES.111.964676

3. Maddox TM, Borden WB, Tang F, et al. Implications of the 2013 ACC/AHA cholesterol guidelines for adults in contemporary cardiovascular practice. J Am Coll Cardiol. 2014;64(21):2183-2192. doi:10.1016/j.jacc.2014.08.041

4. Lin I, Sung J, Sanchez RJ, et al. Patterns of statin use in a real-world population of patients at high cardiovascular risk. J Manag Care Spec Pharm. 2016;22(6):685-698. doi:10.18553/jmcp.2016.22.6.685

5. Wang WT, Hellkamp A, Doll JA, et al. Lipid testing and statin dosing after acute myocardial infarction. J Am Heart Assoc. 2018;7(3). doi:10.1161/JAHA.117.006460

6. Olufade T, Zhou S, Anzalone D, et al. Initiation patterns of statins in the 2 years after release of the 2013 American College of Cardiology/ American Heart Association (ACC/AHA) cholesterol management guideline in a large US health plan. $J$ Am Heart Assoc. 2017;6(5). doi:10.1161/JAHA.116.005205

7. Bellows BK, Olsen CJ, Voelker J, Wander C. Antihyperlipidemic medication treatment patterns and statin adherence among patients with ASCVD in a managed care plan after release of the $2013 \mathrm{ACC} /$ AHA guideline on the treatment of blood cholesterol. J Manag Care Spec Pharm. 2016;22(8):892-900. doi:10.18553/jmcp.2016.22.8.892

8. Tran JN, Kao TC, Caglar T, et al. Impact of the 2013 cholesterol guideline on patterns of lipid-lowering treatment in patients with atherosclerotic cardiovascular disease or diabetes after 1 year. J Manag Care Spec Pharm. 2016;22(8):901-908. doi:10.18553/ jmcp.2016.22.8.901

9. Okerson T, Patel J, DiMario S, Burton T, Seare J, Harrison DJ. Effect of 2013 ACC/AHA blood cholesterol guidelines on statin treatment patterns and low-density lipoprotein cholesterol in atherosclerotic cardiovascular disease patients. $J$ Am Heart Assoc. 2017;6(3). doi:10.1161/JAHA.116.004909
10. Pokharel Y, Tang F, Jones PG, et al. Adoption of the 2013 American College of Cardiology/American Heart Association cholesterol management guideline in cardiology practices nationwide. JAMA Cardiol. 2017;2(4):361. doi:10.1001/jamacardio.2016.5922

11. Brown TM, Deng L, Becker DJ, et al. Trends in mortality and recurrent coronary heart disease events after an acute myocardial infarction among Medicare beneficiaries, 2001-2009. Am Heart $J$. 2015;170(2):249-255.e2. doi:10.1016/j.ahj.2015.04.027

12. Levitan EB, Muntner P, Chen L, et al. Burden of coronary heart disease rehospitalizations following acute myocardial infarction in older adults. Cardiovasc Drugs Ther. 2016;30(3):323-331. doi:10.1007/s10557-016-6653-6

13. Faurot KR, Jonsson Funk M, Pate V, et al. Using claims data to predict dependency in activities of daily living as a proxy for frailty. Pharmacoepidemiol Drug Saf. 2015;24(1):59-66. doi:10.1002/ pds.3719

14. R Core Team. R: A Language and Environment for Statistical Computing. Vienna, Austria: $\mathrm{R}$ Foundation for Statistical Computing; 2018.

15. Virani SS, Pokharel Y, Steinberg L, et al. Provider understanding of the 2013 ACC/AHA cholesterol guideline. J Clin Lipidol. 2016;10 (3):497-504.e4. doi:10.1016/j.jacl.2015.11.002

16. Jacobson TA, Ito MK, Maki KC, et al. National lipid association recommendations for patient-centered management of dyslipidemia: part 1 - full report. J Clin Lipidol. 2015;9(2):129-169. doi:10.1016/j. jacl.2015.02.003

17. Pokharel Y, Akeroyd JM, Virani SS. Cholesterol guidelines: more similar than different. Prog Cardiovasc Dis. 2016;59(2):190-199. doi:10.1016/j.pcad.2016.07.010

18. Grundy SM, Stone NJ, Bailey AL, et al. 2018 AHA/ACC/AACVPR/ AAPA/ABC/ACPM/ADA/AGS/APhA/ASPC/NLA/PCNA guideline on the management of blood cholesterol: a report of the American College of Cardiology/American Heart Association task force on clinical practice guidelines. J Am Coll Cardiol. 2019;73(24):e285350. doi:10.1016/j.jacc.2018.11.003

19. Cannon CP, Blazing MA, Giugliano RP, et al. Ezetimibe added to statin therapy after acute coronary syndromes. $N$ Engl $J$ Med. 2015;372(25):2387-2397. doi:10.1056/NEJMoa1410489

20. Sabatine MS, Giugliano RP, Keech AC, et al. Evolocumab and clinical outcomes in patients with cardiovascular disease. $N$ Engl J Med. 2017;376(18):1713-1722. doi:10.1056/NEJMoa1615664

21. Schwartz GG, Steg PG, Szarek M, et al. Alirocumab and cardiovascular outcomes after acute coronary syndrome. $N$ Engl $\mathrm{J} \mathrm{Med.}$ 2018;379(22):2097-2107. doi:10.1056/NEJMoa1801174
Clinical Epidemiology

\section{Publish your work in this journal}

Clinical Epidemiology is an international, peer-reviewed, open access, online journal focusing on disease and drug epidemiology, identification of risk factors and screening procedures to develop optimal preventative initiatives and programs. Specific topics include: diagnosis, prognosis, treatment, screening, prevention, risk factor modification,

Submit your manuscript here: https://www.dovepress.com/clinical-epidemiology-journal systematic reviews, risk \& safety of medical interventions, epidemiology \& biostatistical methods, and evaluation of guidelines, translational medicine, health policies \& economic evaluations. The manuscript management system is completely online and includes a very quick and fair peer-review system, which is all easy to use. 